\title{
Radioterapia guiada por imagem: técnicas e controle de qualidade
} Image-guided radiation therapy: techniques and quality control

\author{
Laura Furnari1 $\odot$, Leandro R. Fairbanks ${ }^{2} \odot$ \\ ${ }^{1}$ Radioterapia, Instituto de Radiologia, Hospital das Clínicas, Faculdade de Medicina da \\ Universidade de São Paulo, São Paulo, Brasil \\ ${ }^{2}$ Radioterapia, Américas Centro de Oncologia Integrado, Niterói, Brasil
}

\begin{abstract}
Resumo
A radioterapia é uma modalidade de tratamento em constante evolução que procura atingir o seguinte objetivo: irradiar, com precisão cada vez maior, o alvo, poupando ao máximo as estruturas vizinhas. Para alcançar esse objetivo em tratamentos complexos como radioterapia de intensidade modulada (IMRT), radiocirurgia estereotática (SRS) e radioterapia estereotática de corpo (SBRT), surgiu a radioterapia guiada por imagem (image guided radiotherapy — IGRT). Neste artigo, são descritos os princípios de funcionamento e as características dos seguintes principais dispositivos utilizados: Electronic portal imaging, Cone beam, ExacTrac e Calypso. Como todo o sistema que executa procedimentos que envolvem pacientes, os dispositivos de IGRT também precisam ser submetidos a testes de controle de qualidade que garantam a segurança dos pacientes e a precisão dos tratamentos. Para os diferentes dispositivos apresentamos testes com foco na geometria, na qualidade da imagem, no sistema de operação e na segurança. Também são apresentadas a frequência recomendada e a tolerância aceitável.
\end{abstract}

Palavras-chave: radioterapia; controle de qualidade; radioterapia guiada por imagem.

\begin{abstract}
Radiation therapy is a constantly evolving treatment modality seeking to achieve the following goal: to irradiate with increasing precision the target, saving the neighboring structures to the maximum extent. To achieve this goal in complex treatments such as intensity-modulated radiation therapy (IMRT), stereotactic radiosurgery (SRS) and stereotactic body radiation therapy (SBRT), image-guided radiation therapy (IGRT) has emerged. This article describes the operating principles, features of the following main devices used: Electronic portal Imaging, Cone beam, ExacTrac and Calypso. Like any system that performs procedures involving patients, IGRT devices also need to undergo quality control tests to ensure patient safety and accuracy of treatments. For the different devices tests with focus on geometry, image quality, operating system and safety are presented. The recommended frequency and acceptable tolerance are also given.
\end{abstract}

Keywords: radiotherapy; quality control; image guided radiotherapy.

\section{Introdução}

IGRT, acrônimo de image guided radiotherapy, radioterapia guiada por imagem, tem como principais aplicações os tratamentos que requerem extrema precisão de localização, como radiocirurgia estereotática (SRS), radioterapia estereotática de corpo (SBRT) e radioterapia de intensidade modulada (IMRT).

A IGRT foi um passo importante na evolução tecnológica da radioterapia, pois trouxe a possibilidade de uma verificação mais cuidadosa da região a ser irradiada, com dispositivos como ultrassom, tomografia computadorizada (CT) de kilo ou mega voltagem ( $\mathrm{kV}$ ou MV), Cone beam kV ou MV e alta frequência realizados diariamente na sala de tratamento antes do procedimento radioterápico. As imagens tridimensionais de alta resolução obtidas são comparadas, utilizando um software especial de comparação, àquelas de referência realizadas durante o processo de planejamento. O sistema calcula então o deslocamento ocorrido e envia instruções para a mesa de tratamento, que se move para levar o alvo para a posição correta. Em alguns casos são implantados marcadores no tumor para ajudar a visualização do movimento dele.

O conjunto diversificado de ferramentas de imagem tornou possível lidar tanto com o movimento interfracionário, mudanças na posição do alvo ao longo do tempo entre as frações, como com o movimento intrafracionário, isto é, o movimento do órgão durante a sessão do tratamento, geralmente em razão da respiração ou de outros processos fisiológicos.

A IGRT conduziu à radioterapia adaptativa, que consiste na modificação do planejamento, durante o curso de sua 
aplicação, quando são observadas alterações importantes, seja no tamanho seja na localização do tumor.

Pode-se resumir os objetivos da IGRT em:

- exatidão: verificar o posicionamento do alvo;

- precisão: na definição das margens do planning target volume (PTV);

- adaptação às alterações durante o tratamento: corrigir e reduzir os erros de setup, estimar as alterações anatômicas e fazer um replanejamento.

Como as imagens de IGRT são obtidas diariamente, um importante aspecto a ser considerado é a dose que o paciente recebe nessas verificações.

As críticas que podem ser apontadas para a IGRT são o aumento da complexidade, o surgimento de novas fontes de erro, o aumento da dose nos pacientes e da carga de trabalho e a necessidade de mais recursos de infraestrutura; porém, apesar dessas vulnerabilidades, deve-se reconhecer o valor dos sistemas de IGRT como ferramentas que aumentam a precisão de processos já em uso.

\section{Dispositivos de Image Guided Radiotherapy}

Os principais dispositivos usados atualmente para produzir imagens de IGRT são:

- Electronic portal imaging;

- Cone beam;

- ExacTrac;

- Calypso.

\subsection{Electronic Portal Imaging}

O electronic portal imaging (EPID) é um painel plano, constituído de uma placa de silício amorfo (AmSi), acoplado ao acelerador rotacionando, juntamente com o cabeçote, de forma manual ou automática, empregando um braço robótico triarticulado. O paciente é posicionado entre o cabeçote e o EPID.

A radiação é convertida em luz por meio da placa cintiladora de silício, e pode ser detectada de duas maneiras: por meio de uma matriz de fotodiodos colocados abaixo da placa ou por meio de câmeras fotográficas que capturam as imagens do padrão produzido na placa. Em ambos os sistemas, obtêm-se quadros em intervalos de tempo regulares, e a imagem final é a média dos quadros. Desse modo, gera-se o mapa de fluências que incide sobre ele e, com isso, tem-se uma medida relativa de dose. O EPID tornou-se o sistema padrão para aquisição de imagens portais em aceleradores lineares.

O sinal detectado pelo EPID é expresso em unidades de calibração (CU) e, ao se fazer uma correlação entre as medidas desse portal e uma dosimetria feita nas mesmas condições com uma câmara de ionização, obtém-se a dose absoluta incidente sobre o EPID. Uma das principais aplicações é usar esse painel na verificação dosimétrica absoluta para liberação de tratamentos com campos dinâmicos, tipo IMRT. Assim, além de sua mais importante função, a de gerar imagens dos pacientes, os EPID, hoje em dia, são amplamente utilizados em verificações da garantia de qualidade e da dosimetria de planejamentos. Outras atividades importantes são a dosimetria in vivo e as aplicações em dosimetria tridimensional.

As grandes vantagens do EPID sobre os sistemas usados anteriormente são a linearidade, a estabilidade, a alta resolução e a flexibilidade.

A imagem do tumor obtida no EPID é comparada à imagem reconstruída digitalmente (digitally reconstructed radiography - DRR), oriunda do planejamento. Assim, determinam-se os deslocamentos necessários na mesa de tratamento para se fazer coincidir as imagens, o que ocorre quando o paciente está posicionado corretamente. Se a imagem do tumor obtida no EPID estiver deformada em relação àquela da DRR e o deslocamento indicado pelo software não for suficiente para que as imagens coincidam, é necessário modificar o planejamento, ou seja, fazer radioterapia adaptativa.

\subsection{Cone Beam}

São dispositivos compostos de dois braços robóticos, com uma fonte de raios $X$ num braço e um painel detector de AmSi no outro. São utilizados para aquisição de imagens digitais dos pacientes na mesa de tratamento, com tensões da ordem de raios X diagnóstico, de 50 a $125 \mathrm{kV}$. Executam uma tomografia na máquina de tratamento, gerando imagens volumétricas que possibilitam a visualização dos tecidos moles, permitindo, assim, a verificação do formato e a posição dos órgãos e do volume-alvo, bem como a observação da mudança da anatomia do paciente durante o tratamento. Essas imagens fornecem informações mais explícitas que as imagens obtidas com MV, além de terem a vantagem de entregar uma dose menor. Marcadores radiopacos implantados servem como guias de referência para a localização, quando é feita a comparação com as imagens da tomografia de simulação. Há, porém, uma diferença entre essas imagens, porque aquela obtida no acelerador linear é uma tomografia por feixe cônico Cone beam computed tomography (CBCT) - , produzida com feixe em formato de cone, enquanto a imagem da CT de simulação é produzida por um feixe colimado e estreito em formato de leque.

Os filtros bowtie são muito aplicados no CBCT para modular a emissão da fonte de radiação. O termo bowtie aplica-se a uma classe de filtros com formato simétrico bilateral cuja espessura aumenta com a distância a partir do centro. Esse filtro compensa a diferença no comprimento do caminho do feixe por meio do plano axial do objeto, de modo que uma fluência mais uniforme possa ser entregue no detector. O risco de overflow nos elementos periféricos do detector é diminuído com esses filtros, o que reduz a exigência no range dinâmico do detector e permite melhor detectabilidade do contraste. Esses filtros também reduzem o espalhamento, a maior causa dos artefatos na imagem. Uma grande unidade de CBCT, usada em IGRT, geralmente 
tem diferentes filtros "bowtie" intercambiáveis para diferentes configurações de exposição, mas em sistemas com field of view (FOV) relativamente pequeno, seus benefícios não são muito grandes.

Os principais sistemas comerciais são o $\mathrm{OBI}^{\circledR}$, da Varian, o XVI ${ }^{\circledR}$, da Elekta, e o In-Line KVision ${ }^{\circledR}$, da Siemens.

Também existe a CT em trilhos, que consiste em um scanner na mesma sala do acelerador. A maca do paciente pode ser rotacionada em $180^{\circ}$ para transferir o paciente do acelerador para a CT.

O cone beam de MV é usado em máquinas de tomoterapia, nas quais o feixe de tratamento também é helicoidal.

\subsection{ExacTrac}

O ExacTrac ${ }^{\oplus}$, da BrainLab, é um sistema constituído principalmente de dois subsistemas: um sistema de rastreamento por infravermelho (IR) e um sistema de raios $X$ de quilovoltagem. O primeiro contém câmeras fixadas no teto, que emitem raios $I R$ e detectam os raios refletidos em marcadores fiduciais externos fixados no paciente, na mesa de tratamento, ou em acessórios de localização. No segundo subsistema, dois tubos de raios $X$ estão embutidos no chão da sala de tratamento e duas telas de detectores de AmSi estão fixadas no teto. Quando acionados, fornecem duas imagens radiográficas oblíquas do paciente.

A forma de proceder é posicionar o paciente com auxílio dos lasers, verificar se os marcadores estão sendo detectados pelas duas câmeras, adquirir duas imagens radiográficas com os tubos 1 e 2 e ajustar seu contraste e fazer a fusão automática das imagens com a DRR. Avalia-se, com as ferramentas de sobreposição, se a fusão está aceitável, e observam-se os valores indicados de deslocamentos rotacional e translacional da mesa, que são os que fornecem a máxima similaridade com a DRR. Esses deslocamentos são realizados e, finalmente, repete-se a aquisição das imagens para confirmar o correto posicionamento do paciente.

O CyberKnife $\mathrm{VSI}^{\circledR}$ tem um modo de funcionamento semelhante ao do ExacTrac da BrainLab: possui duas unidades $\mathrm{kV}$ estereotáticas no teto para produzir imagens captadas por dois detectores planares localizados no chão, e tem marcadores externos que são rastreados usando câmaras esteroscópicas. No sistema CyberKnife VSI ${ }^{\oplus}$, usam-se marcadores fiduciais implantados, o que permite ao sistema construir um modelo de correlação que é usado para alterar a liberação da radiação por meio de um rastreamento robótico do movimento do tumor no próprio acelerador. Se um desalinhamento é percebido, o sistema interrompe a radiação, aplica automaticamente um deslocamento na mesa de tratamento e permite que o tratamento prossiga. Dependendo do ângulo do gantry, mais imagens de $\mathrm{kV}$ podem ser obtidas a fim de melhorar a precisão do tratamento.

\subsection{Calypso}

O sistema Calypso ${ }^{\oplus}$ GPS for the Body, da Varian Medical Systems, é um dispositivo de rastreamento que monitora a posição de três transponders eletromagnéticos de $8 \mathrm{~mm}$ de comprimento e $2 \mathrm{~mm}$ de diâmetro implantados no tumor. Os transponders transmitem a localização do alvo, 25 vezes por segundo, ao sistema de rastreamento do Calypso, por meio de ondas de radiofrequência. Ele alerta quando o alvo está fora dos limites aceitáveis e interrompe o feixe, permitindo correções durante o tratamento.

Os transponders também podem ser usados na superfície do paciente quando é necessário monitorar seus movimentos. Antes do tratamento, os pacientes são submetidos a um procedimento ambulatorial simples, no qual os transponders são implantados no tumor sob a orientação do ultrassom de maneira semelhante a uma biópsia por agulha, ou simplesmente colocados sobre a superfície da pele.

A matriz de detecção é colocada acima do paciente e é rastreada com três câmeras ópticas referenciadas ao isocentro da sala.

Comparando-se as posições dos centroides dos transponders obtidas no início da sessão de tratamento com aquelas na CT de planejamento pode-se alinhar o paciente em relação ao movimento interfração. Durante a administração do tratamento, o centroide dos transponders implantados é monitorado "continuamente", permitindo as correções dos movimentos intrafração.

O monitoramento do Calypso possibilita reduzir as margens entre o volume do clinical target volume (CTV) e o do PTV, pois garante que a dose prescrita está sendo administrada precisamente, o que diminui o número de erros geométricos no tratamento.

Algumas limitações dessa metodologia incluem a invasividade do procedimento, o custo do transponder, a falta de informação sobre tecidos moles e artefatos de ressonância magnética no acompanhamento pós-tratamento.

O Calypso, apesar de ser um dispositivo de IGRT, não gera uma imagem, e sim realiza o rastreamento do paciente a partir das informações em radiofrequências dos transponders. Assim, ele não contribui com nenhuma dose no paciente.

\section{Controle da Qualidade}

Alguns testes de garantia da qualidade dos sistemas de IGRT utilizam o sistema de lasers da sala de tratamento como referência do isocentro do acelerador linear. Dessa forma, antes da realização desses testes, é de extrema importância garantir a precisão do sistema de lasers.

\subsection{Electronic Portal Imaging1-3}

\subsubsection{Funcionalidade}

A análise da funcionalidade do sistema inicia-se com a verificação da exportação de dados com a utilização de um paciente teste. Em seguida, adquire-se a imagem de um objeto para uma verificação geral, e avalia-se o funcionamento do braço robótico do EPID estendendo-o e 
recolhendo-o pelo console e/ou pelo controle interno na sala de tratamento.

\subsubsection{Sistema Anticolisão}

O braço robótico do EPID possui dispositivos anticolisão. Para verificar a funcionalidade desse sistema, o braço deve ser estendido e o dispositivo anticolisão acionado enquanto ele estiver em movimento. O teste é bem-sucedido se o braço, o gantry e a mesa pararem imediatamente. O alarme sonoro, então, será acionado e o sistema gerará um intertravamento de colisão. A periodicidade do teste é diária, variando sempre o dispositivo anticolisão a ser acionado.

\subsubsection{Precisão do Posicionamento}

O EPID deve ser estendido para uma distância vertical conhecida e a capa de proteção precisa ser retirada. Com o auxílio da ponteira calibrada e de um instrumento de medida, verifica-se o posicionamento vertical do EPID. O mesmo procedimento é realizado para os movimentos longitudinal e lateral do EPID. A diferença entre o valor medido e o valor nominal de deslocamento não deve exceder $2 \mathrm{~mm}$.

\subsubsection{Precisão do Isocentro}

Para a verificação da precisão do isocentro do EPID, o fabricante fornece o objeto simulador cube phantom (Figura 1A). Alinha-se o cube phantom sobre a mesa de tratamento, utilizando os lasers de posicionamento da sala, e realizam-se imagens ortogonais $\left(0^{\circ}, 90^{\circ}, 180^{\circ}\right.$ e $270^{\circ}$ ). Deve-se verificar se o retículo digital do software de análise está dentro dos limites da esfera para as quatro imagens, atentando-se ao limite de tolerância de $2 \mathrm{~mm}$.

\subsubsection{Resolução e Contraste}

Incluído em seu equipamento, os fabricantes dos aceleradores lineares disponibilizam o Las Vegas Phantom (CDP) (Figura 1B). O teste consiste em posicionar o CDP sobre a mesa de tratamento e adquirir imagens utilizando todas as energias, taxas de doses e protocolos de imagens que são utilizados na rotina clínica para verificar a quantidade de círculos que pode ser visualizada. Esse teste deve ser realizado durante o comissionamento do equipamento, e o resultado é utilizado como referência para as imagens realizadas mensalmente.

\subsubsection{Uniformidade}

Ao irradiar o EPID em um campo homogêneo, a imagem gerada deve se manter uniforme em toda sua extensão, conforme as características do feixe incidente. A avaliação é feita por meio de uma exposição que cubra toda a parte sensível do detector, verificando-se a uniformidade da imagem gerada e comparando-a com a imagem realizada no comissionamento do equipamento.

\subsection{Cone Beam Computed Tomography4-6}

\subsubsection{Funcionalidade, Sistema Anticolisão e Precisão do Posicionamento}

Os testes de funcionalidade, sistema anticolisão e precisão do posicionamento para o CBCT seguem o mesmo protocolo do EPID, já citado.

\subsubsection{Precisão no Isocentro do Cone Beam Compu- ted Tomography e Acurácia do Movimento Remoto da Mesa}

Para a realização desse teste, é necessária uma imagem tomográfica do objeto simulador marker phantom (Figura 2A).
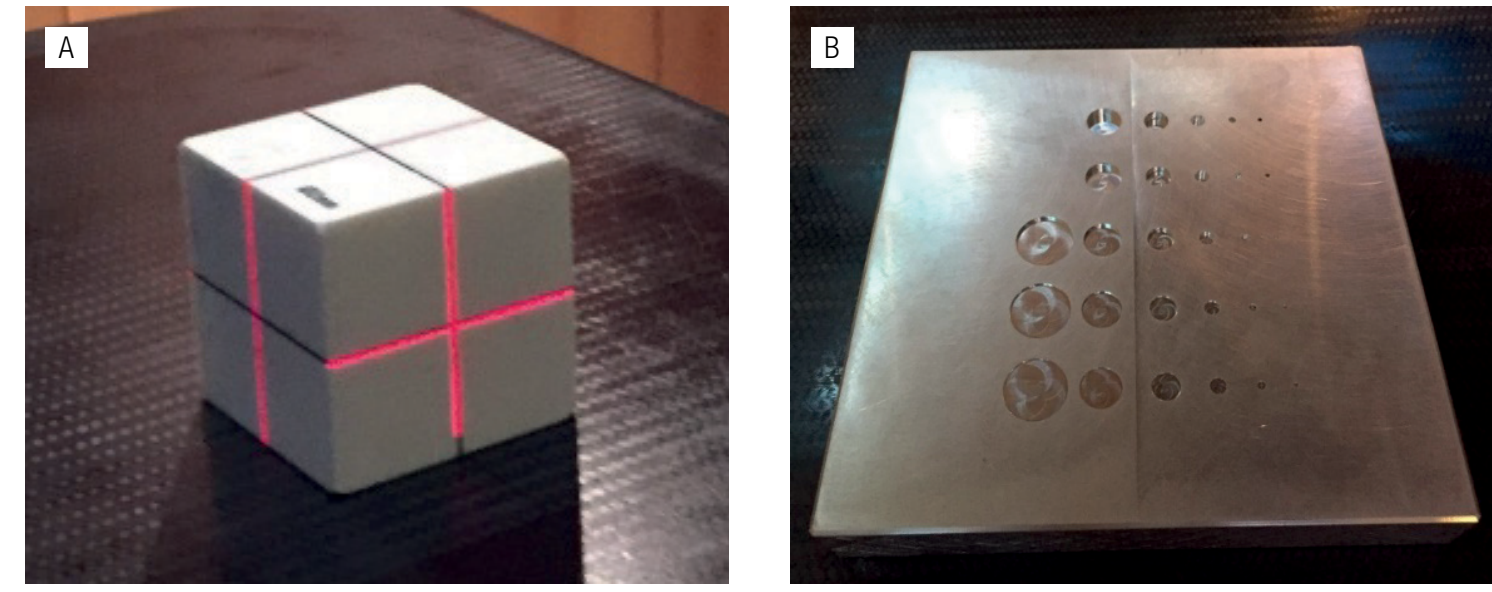

Figura 1 - 0bjetos simuladores utilizados nos controles de qualidade do electronic portal imaging: (A) Cube Phantom, cubo em água sólida contendo em seu centro uma esfera radiopaca de $2 \mathrm{~mm}$ de diâmetro; e (B) Las Vegas Phantom, bloco de alumínio com círculos em baixo relevo contendo diâmetros que variam de 0,5 a $15 \mathrm{~mm}$, profundidade de 0,5 a $4,5 \mathrm{~mm}$ e contrastes de 0,6 a $5,1 \%$, para $6 \mathrm{MV}$, e de 0,4 a $3,4 \%$, para $15 \mathrm{MV}$. 
Primeiramente, posiciona-se o objeto simulador sobre a mesa do acelerador, alinhando-o com os lasers da sala de tratamento, para, em seguida, realizar deslocamentos predeterminados em todas as direções dos eixos de coordenadas ( $\mathrm{x}, \mathrm{y}$ e z). Após os deslocamentos, é realizada uma imagem de CBCT para ser comparada com a CT de referência. Os valores de deslocamentos sugeridos pelo sistema devem coincidir com os realizados no objeto simulador. Após a movimentação remota da mesa, os lasers da sala de tratamento deverão estar alinhados novamente com as marcações do objeto simulador. A periodicidade desse teste é diária, e a tolerância não deve ultrapassar 2 mm de distância entre a posição do laser e a marcação do objeto simulador.

Para a realização dos demais controles de qualidade do CBCT, utiliza-se o objeto simulador Catphan ${ }^{\circledR}$ (Figura 2B), fornecido pelo fabricante do acelerador linear, que possui diferentes regiões para diversas avaliações. O usuário deve criar um paciente teste no sistema de planejamento e fazer uma imagem CBCT desse simulador para analisar, na mesma imagem, os testes: linearidade das unidades Hounsfield (HU), uniformidade de HU, resolução espacial, resolução de baixo contraste, linearidade espacial em um corte e espessura de corte. A periodicidade dos controles de qualidade que utilizam o Catphan é mensal, porém, se o usuário julgar os testes como estáveis, pode-se mudar a periodicidade para semestral.

\subsubsection{Linearidade das Unidades Hounsfield}

A linearidade do número de $\mathrm{HU}$ é avaliada com o inserto CTP404 (Figura 3A), que contém seções com diferentes materiais de diferentes densidades e, consequentemente, possui diferentes HU. O manual do Catphan apresenta diversas especificações tabeladas dos materiais, como fórmula química, número atômico efetivo, densidade eletrônica e valores máximos e mínimos de HU, que servirão como referência nas medidas. Utilizando uma região de interesse
(ROI) de $(7 \times 7) \mathrm{mm}^{2}$, mensura-se o valor de $\mathrm{HU}$ para todos os materiais do simulador.

\subsubsection{Uniformidade de Unidades Hounsfield}

Para avaliar a uniformidade de $\mathrm{HU}$, utiliza-se o inserto CTP486 (Figura 3B), que deve ser localizado na CBCT. Com uma ROI de $(1 \times 1) \mathrm{cm}^{2}$, mensura-se o HU em cinco posições: centro, inferior, superior, direita e esquerda (dispostos simetricamente da ROI central). Esses valores são comparados entre si, podendo variar até, no máximo, $40 \mathrm{HU}$.

\subsubsection{Resolução Espacial}

A resolução espacial é analisada no inserto CTP528 (Figura 3C). Uma vez localizada essa seção, deve-se verificar até que padrão é possível diferenciar a separação entre as barras. Pode-se utilizar a ferramenta de zoom e contraste e brilho da imagem para facilitar a visualização. É importante manter o mesmo padrão de análise para as verificações futuras. A tolerância é a distinção até as linhas do grupo 6, que possuem seis pares de linha por centímetro $(\mathrm{Lp} / \mathrm{cm})$.

\subsubsection{Resolução de Baixo Contraste}

Nesse teste, utiliza-se o inserto CTP515 (Figura 3D). Deve-se contar o número de discos visíveis e registrar o diâmetro e o contraste do último disco cuja visualização foi possível. É esperado conseguir visualizar o disco de $7 \mathrm{~mm}$ com $1 \%$ de contraste.

\subsubsection{Linearidade Espacial em um Corte}

A linearidade espacial de um corte também é analisada com o inserto CTP404 (Figura 3A). Após localizá-lo, deve-se utilizar a ferramenta digital de medida para mensurar a distância entre os furos posicionados nos vértices de um quadrado de $5 \mathrm{~cm}$ de lado. As medidas não podem variar mais que $5 \mathrm{~mm}$ do valor correto.

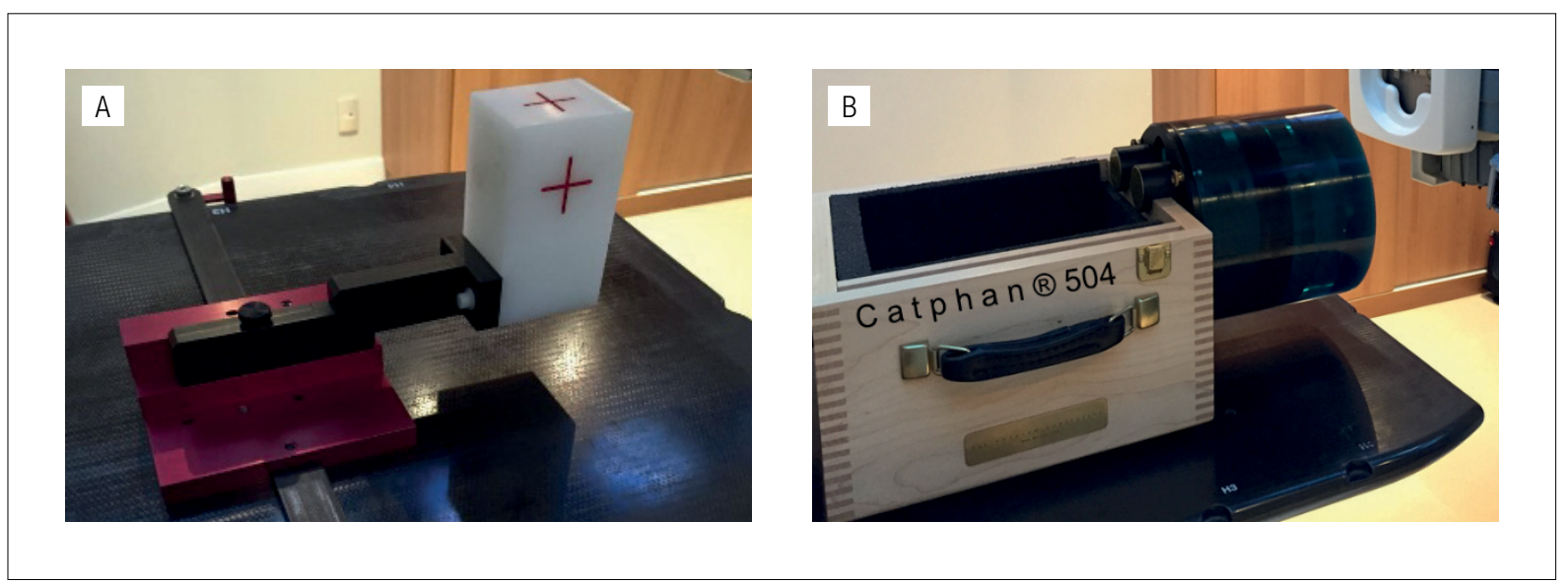

Figura 2 - Objetos simuladores utilizados nos controles de qualidade do Cone beam computed tomography. (A) Marker Phantom, objeto simulador fixo em uma barra de indexação que permite o deslocamento nos eixos de coordenadas $(\mathrm{x}, \mathrm{y}$ e $\mathrm{z}$ ) de 1,5 cm e de 2,0 cm; e (B) Catphan, objeto simulador para controle de qualidade de Cone beam computed tomography com quatro diferentes insertos. 


\subsubsection{Espessura de Corte}

Ainda no inserto CTP404 (Figura 3A), mensura-se a espessura de corte. Inicialmente, determina-se a largura total na metade da intensidade máxima da imagem dos fios (do inglês full width at half maximum - FWHW), ampliando ao máximo a imagem do inserto CTP404 - recomenda-se acertar seu contraste e brilho para melhor visualização. Uma vez ajustada a imagem, a ferramenta de medida é, então, posicionada em uma das extremidades do fio. Ao passar a imagem para o corte posterior ou anterior, posiciona-se a outra extremidade da ferramenta na mesma extremidade do fio - a medida encontrada é a FWHW. Multiplica-se então a FWHW pela tangente de $23^{\circ}(0,42)$ e obtém-se a espessura de corte. A tolerância recomendável é de menos que 5\% da espessura escolhida na aquisição do CBCT.

\subsection{ExacTrac7-11}

\subsubsection{Calibração do Sistema}

$O$ acelerador linear, o sistema de rastreamento por IR e o sistema de raios $X$ possuem coordenadas geométricas independentes, sendo, portanto, necessário correlacioná-las semanalmente.

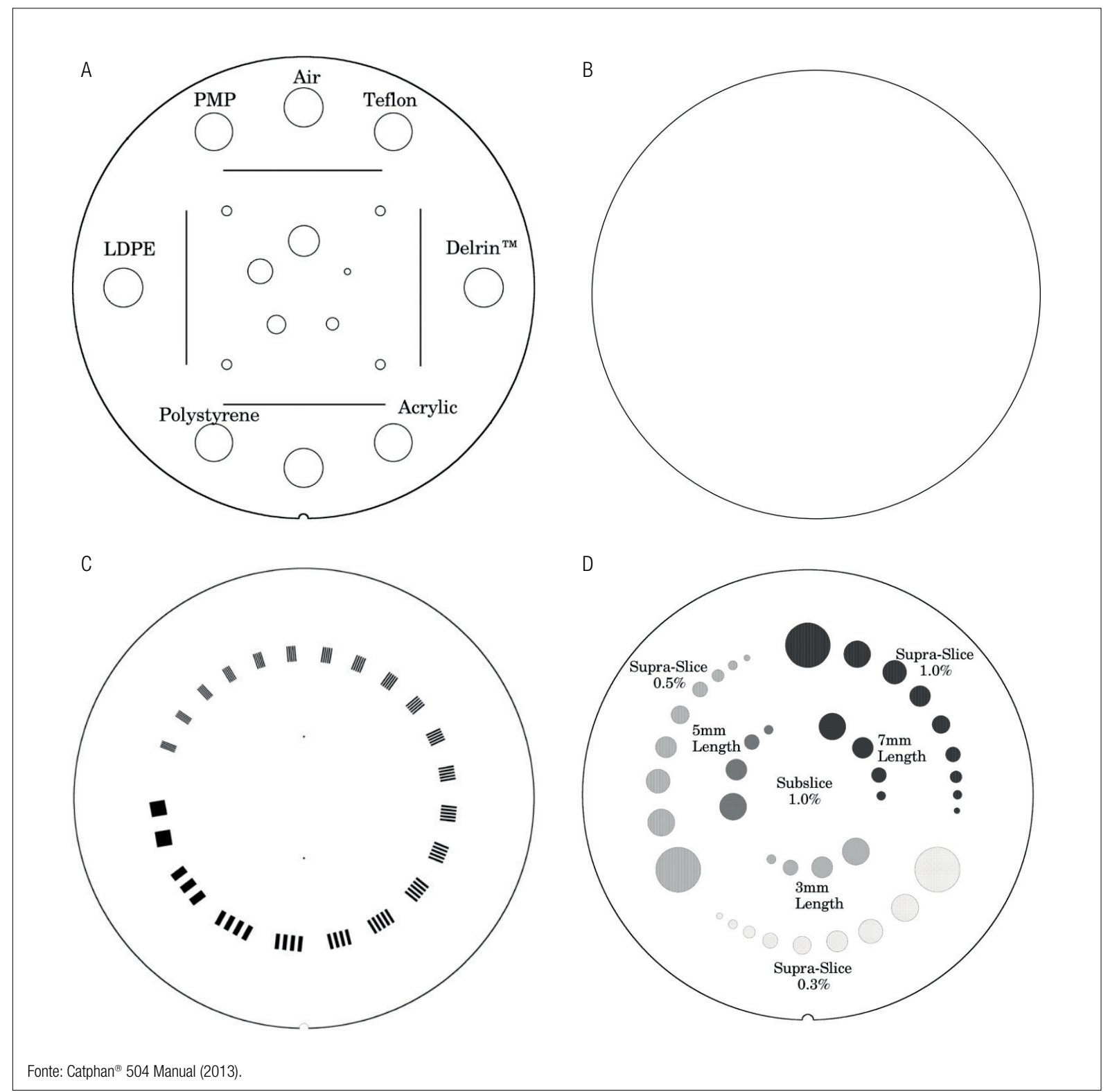

Figura 3 - Insertos do Catphan: (A) CTP404, que possui sete discos com diferentes materiais; quatro furos com $3 \mathrm{~mm}$ de diâmetro, posicionados nos vértices de um quadrado de $5 \mathrm{~cm}$ de lado; e dois pares de fio inclinados de $23^{\circ}$, sendo um par orientado paralelamente ao eixo x e 0 outro, ao eixo y; (B) CTP486, que possui um disco uniforme de $20 \mathrm{~cm}$ de diâmetro; (C) CTP528, que contém padrões em barra de 1 a 21 Lp/cm; (D) CTP515, que contém discos com diferentes contrastes (0,3, 0,5 e 1\%); em cada grupo de contraste, os discos podem possuir nove dimensões diferentes (variando de 2 a $15 \mathrm{~mm}$ para supra-slice) ou quatro dimensões diferentes (variando de 3 a $9 \mathrm{~mm}$ para 0 subslice). 
A primeira correlação é entre as coordenadas do acelerador linear e as do sistema IR. Para essa calibração, deve-se posicionar o objeto simulador ET Isocenter Phantom (Figura 4A) alinhado com os lasers da sala e realizar a calibração seguindo as instruções do software do ExacTrac. Essa calibração servirá de base para a calibração do sistema de raios $X$. Na sequência, para correlacionar as coordenadas do acelerador linear com o sistema de raios $X$, utiliza-se o objeto simulador ET X-Ray Calibration (Figura 4B), que deve ser minuciosamente alinhado com os lasers da sala, já que essa calibração vai influenciar o resultado final da conferência do posicionamento. Após esse alinhamento, rastreia-se a posição do objeto simulador com o sistema de $\mathrm{IR}$, calibrado previamente. Fazem-se então as imagens com o sistema de raios $\mathrm{X}$, e utilizando o software de análise da imagem, deve-se atentar à verificação das cruzes laranjas centradas nos círculos amarelos, ou seja, se os marcadores radiopacos estão centralizados no posicionamento esperado.

Após as calibrações do ExacTrac, o próprio software oferece duas opções de conferência: move isocenter phantom to current isocenter, que verifica se as coordenadas do sistema IR coincidem com as coordenadas do acelerador linear, e verify $X$-Ray calibration, que examina se as coordenadas do sistema de raios $X$ coincidem com as do acelerador linear.

\subsubsection{Precisão da Localização Estática do Sistema de Rastreamento por Infravermelho}

Essa avaliação verifica a precisão do deslocamento da mesa de tratamento pós-verificação com o sistema IR.
Para a realização desse teste, primeiramente alinha-se o objeto simulador com fiduciais refletores utilizando os lasers da sala de tratamento. Em seguida, aplica-se um deslocamento conhecido em uma das direções do eixo de coordenadas e o simulador é reposicionado utilizando o sistema de IR. Verifica-se visualmente pelos lasers se o objeto simulador retornou ao isocentro e se o deslocamento aplicado pela mesa coincide com o deslocamento inicialmente realizado. Esse teste deve ser repetido para todas as direções do eixo de coordenadas ( $x$, y e $z$ ), e para casos de radiocirurgia, o limite deve ser inferior a $1 \mathrm{~mm}$.

\subsubsection{Precisão da Localização Estática do Sistema de Raios $X$}

De maneira análoga, é necessário verificar a precisão do deslocamento da mesa quando se utiliza o sistema de raios $X$. Inicialmente, alinha-se o objeto simulador com marcadores radiopacos utilizando os lasers da sala de tratamento, e faz-se uma imagem com ambos os tubos de raios $X$. Desloca-se o objeto simulador propositalmente com valores predefinidos e fazem-se novas imagens com ambos os tubos de raios $X$. A correção na mesa é aplicada baseada na fusão do software do ExacTrac, e verifica-se visualmente se o objeto simulador está novamente alinhado com os lasers e se os deslocamentos da mesa translacionais e rotacionais coincidem com o deslocamento aplicado no objeto simulador. A tolerância desse teste deve ser igual ou menor que $1 \mathrm{~mm}$, se o sistema for utilizado para casos de radiocirurgia.
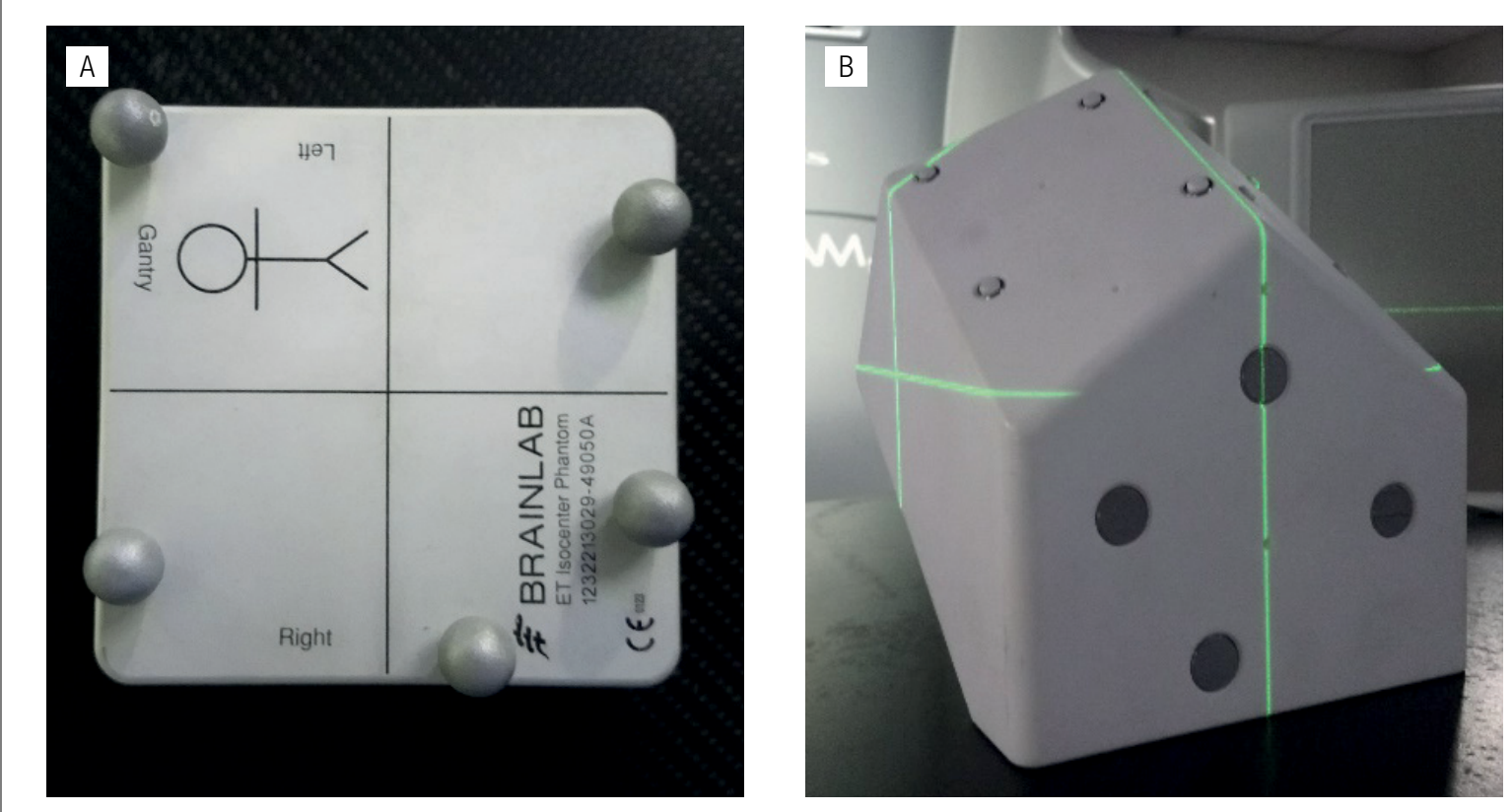

Figura 4 - Objetos simuladores utilizados nos controles de qualidade do ExacTrac: (A) ET Isocenter Phantom, que possui cinco marcadores refletores e impressão de orientação; (B) verify X-Ray calibration, que possui marcadores externos refletores e 16 marcadores radiopacos externos. 


\subsubsection{Qualidade da Imagem}

A acurácia na utilização do ExacTrac depende da qualidade da imagem gerada pelo sistema de raios $X$ uma imagem de baixa qualidade pode resultar em um processo inadequado de fusão com a imagem digital reconstruída, ocasionando uma análise incorreta do visualizador. Para esse teste, o usuário deve selecionar $X$-Ray Correction Image e realizar cinco imagens com cada tubo de raios $\mathrm{X}$, visto que o software do ExacTrac identifica e interpola os pixels defeituosos, criando um offset para imagens futuras. A periodicidade dessa avaliação deve ser mensal.

\subsection{Calypso12,13}

\subsubsection{Controle de Qualidade Diário}

O controle de qualidade diário do sistema Calypso verifica o desempenho do sistema por meio do objeto simulador quality assurance fixture (QAF) (Figura 5A) com transponders beacon fornecidos pelo fabricante. No software do Calypso, seleciona-se a função QA e o gantry é posicionado sob a mesa de tratamento. Alinha-se o objeto simulador utilizando o sistema de lasers da sala de tratamento e, caso necessário, pode-se utilizar as chaves de ajustes localizadas no pé do objeto simulador. O detector eletromagnético é posicionado sobre o objeto simulador, conforme as orientações do software, para que o teste possa ser iniciado ao clicar no botão begin test, no software do Calypso. A análise demora menos de um minuto e fornece os desvios das componentes laterais, longitudinais e verticais do isocentro do objeto simulador em relação ao isocentro calibrado do sistema Calypso, e o isocenter offset na forma de desvios do isocentro do objeto simulador a partir do isocentro calibrado do sistema Calypso. O isocenter offset deve ser menor ou igual a $1,5 \mathrm{~mm}$, se o sistema for utilizado para radiocirurgias.

\subsubsection{Dynamic Edge Gating}

Esse teste serve para verificar diariamente se o sistema consegue detectar a conectividade. Coloca-se o objeto simulador sobre a mesa e inicia-se o rastreamento: usa-se um feixe $(10 \times 10) \mathrm{cm}^{2}$ como campo de tratamento e, durante o feixe, aciona-se o gating test no controlador da Dynamic Gating Interface. Se o sistema estiver com sua funcionalidade normal e o feixe ainda estiver ligado, este continuará ligado e o sistema confirmará e exibirá os resultados de teste de gating Beam Hold como CONFIRMED, indicando o funcionamento correto da conectividade de ponta a ponta. Se o feixe estiver desligado, ao pressionar o botão gating test, o sistema não confirma que o feixe foi mantido, e será exibido o resultado do teste Beam Hold como NOT CONFIRMED.

\subsubsection{Calibração do Sistema}

Para iniciar a calibração do sistema, seleciona-se a função calibrate no software do Calypso e alinha-se o objeto simulador isocenter calibration fixture (ICF) (Figura 5B) com o laser da sala de tratamento - se necessário, utiliza-se o ajuste fino no pé do objeto simulador. Adquirem-se duas imagens ortogonais (anterior e lateral) do objeto simulador, certificando-se que seu isocentro e o isocentro do acelerador linear coincidam. Se o limite de tolerância entre os isocentros for respeitado, o teste de alvos óticos é executado, utilizando as três câmeras de IR que definem o quadro de coordenadas de referência do

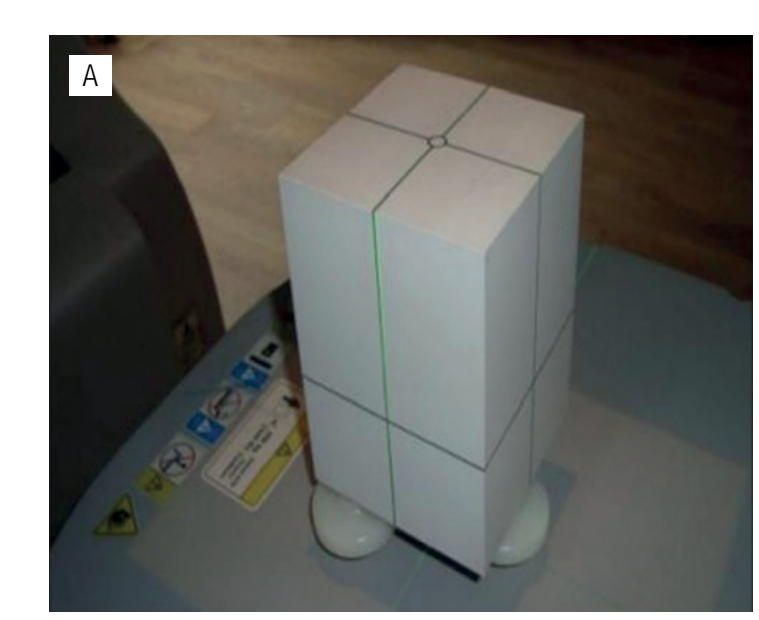

Fonte: Muralidhar et al. ${ }^{12}$

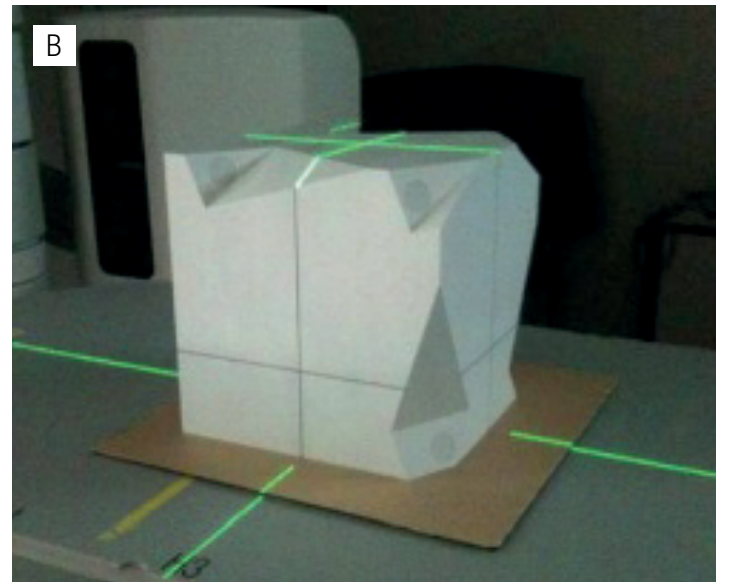

Figura 5 - 0bjetos simuladores utilizados nos controles de qualidade do Calypso: (A) QAF, objeto simulador que contém três Beacon transponders e três "pés" com chave deslizante que permitem ajustar seu alinhamento; (B) ICF, que contém três Beacon transponders, linhas de marcação para permitir 0 alinhamento com os lasers na sala de tratamento, seis refletores óticos, três "pés" com chave deslizante para ajustar 0 alinhamento e uma esfera de tungstênio interna posicionada no isocentro. 
subsistema localização ótica. Ajusta-se o posicionamento do detector eletromagnético e o sistema faz uma autoanálise, apresentando os valores dos desvios dos componentes laterais, longitudinais e verticais do isocentro do sistema Calypso a partir da posição do isocentro da máquina, além do valor isocenter offset, que deve ser menor ou igual a $1 \mathrm{~mm}$.

\subsubsection{Calibração das Câmeras}

A calibração das câmeras deverá ser feita somente se a calibração do sistema não for bem-sucedida. Esse controle de qualidade utiliza dois acessórios: um com formato em $L$ (Figura $6 \mathrm{~A}$ ) e outro com formato em $T$ (Figura 6B). Para a calibração, clica-se no botão calibrate e inicia-se a função camera calibration. O acessório com estrutura em $L$ é posicionado sobre a mesa de tratamento, seguindo a orientação fornecida pelo software do Calypso, e após o alinhamento com os lasers da sala de tratamento, deve-se conferir a detecção correta dos alvos pelas câmeras IR. O teste no software pode ser, então, iniciado e fará uma autoanálise: caso aprovado, recomenda-se remover o acessório em $L$ e prosseguir para o teste com o acessório em T. Caso reprovado, os procedimentos descritos anteriormente devem ser repetidos.

$\mathrm{Na}$ sequência desse teste, o software irá solicitar a seleção do acessório com estrutura em T utilizado na calibração. Posiciona-se a mesa de tratamento de modo que esta fique fora do campo de visão das câmeras IR e rotaciona-se o gantry de forma que este fique sob a mesa de tratamento. Assim, o teste no software do Calypso pode ser iniciado, segurando o acessório em $\mathrm{T}$ e movendo-o em pequenos movimentos circulares ao longo do campo de visão das câmeras em cada uma das três orientações, com as esferas reflexivas posicionadas de maneira oposta ao acelerador linear. O sistema avalia o teste e apresenta como resultado "aprovado" ou "reprovado". Sua finalidade é calibrar as três câmeras independentemente de sua posição e orientação específicas na sala de tratamento. É importante atentar-se para que nenhuma câmera detecte fontes de reflexão dispersa, pois podem interferir no resultado final da calibração.

\section{Conclusão}

Um grande problema com o qual o físico médico pode se deparar atualmente é a velocidade com que as tecnologias se desenvolvem. A IGRT é um típico exemplo disso, pois, com os dispositivos sofisticados de que dispõe, pretende controlar o local da irradiação com precisão submilimétrica, não obstante os movimentos fisiológicos que ocorrem durante a sessão de tratamento. Esse objetivo, porém, só pode ser alcançado se o uso desses recursos for feito de forma controlada e precisa, ou seja, se periodicamente for executado seu controle de qualidade. No presente artigo, foram descritos, para cada um dos principais dispositivos, testes com foco na geometria, na qualidade da imagem, no sistema de operação e na segurança.

\section{Agradecimentos}

Os autores agradecem aos editores o honroso convite.

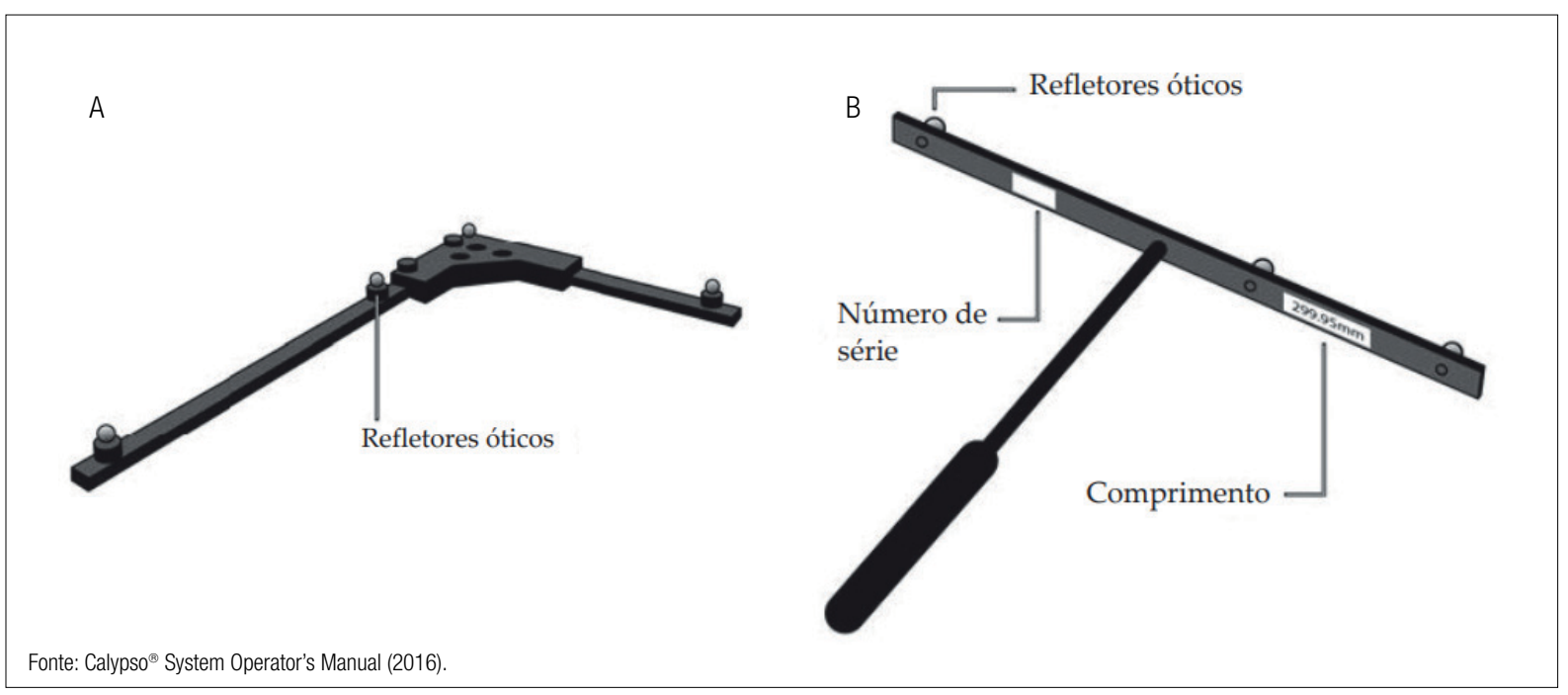

Figura 6 - Objetos simuladores utilizados na calibração das câmeras do Calypso: (A) acessório com estrutura em L, que possui quatro refletores óticos esféricos; e (B) acessório com estrutura em T, que possui três refletores óticos esféricos na respectiva barra transversal e uma alça almofadada. 


\section{Referências}

1. Low DA, Klein EE, Maag DK, Umfleet WE, Purdy JA. Commissioning and periodic quality assurance of a clinical electronic portal imaging device. Int J Radiat Oncol Biol Phys. 1996;34(1):117-23. https://doi. org/10.1016/0360-3016(95)02096-9

2. Herman MG, Kruse JJ, Hagness CR. Guide to clinical use of electronic portal imaging. J Appl Clin Med Phys. 2000;1(2):38-57. https://doi. org/10.1120/1.308249

3. Skrzyński W, Reilly JA, Thwaites ID, Bulski W. EPID image quality: Las Vegas phantom as an objective tool. In: Anais do 13th Congress of the Polish Society of Medical Physics; 2005. Varsóvia; 2005.

4. Yoo S, Kim GG-Y, Hammoud R, Elder E, Pawlicki T, Guan H, et al. A quality assurance program for the On-Board Imager. Med Phys. 2006;33(11):443147. https://doi.org/10.1118/1.2362872

5. Saw CB, Yang Y, Li F, Yue N, Ding C, Komanduri K, et al. Performance Characteristics and Quality Assurance Aspects of Kilovoltage ConeBeam CT on Medical Linear Accelerator 2007;32(2):80-5. https://doi. org/10.1016/j.meddos.2007.01.003
6. The Phantom Laboratory. Catphan ${ }^{\circledast} 504$ Manual. Nova York: The Phantom Laboratory.

7. BrainLab. Clinical User Guide, ExacTrac ${ }^{\oplus}$. Munique: BrainLab.

8. Iftimia I, Halvorsen P. Development of clinically relevant QA procedures for the BrainLab ExacTrac imaging system. J Appl Clin Med Phys. 2018;19(3):108113. https://doi.org/10.1002/acm2.12301

9. Kim J, Wen N, Jin J-Y, Walls N, Kim S, Li H, et al. Clinical commissioning and use of the Novalis Tx linear accelerator for SRS and SBRT. J Appl Clin Med Phys. 2012:13(3):3729. https://doi.org/10.1120/jacmp.v13i3.3729

10. Willoughby T, Lehmann J, A Bencomo J, Jani S, Santanam L, Sethi A, et al. Quality assurance for nonradiographic radiotherapy localization and positioning systems: Report of Task Group 147. Med Phys. 2012;39(4):1728-47.

11. Vinci J. Accuracy of cranial coplanar beam therapy with Brain LAB ExacTrac image guidance [tese]. 2007.

12. Muralidhar KR, Komanduri K, Kumar Rout B, Ramesh KKD. Commissioning and quality assurance of Calypso four-dimensional target localization system in linear accelerator facility. J Med Phys. 2013;38(3):143-7. https://doi.org/10.4103/0971-6203.116376

13. Varian Medical System. Calypso ${ }^{\circledast}$ System Operator's Manual. Palo Alto: Varian Medical System. 